Article

\title{
Bosutinib Inhibits EGFR Activation in Head and Neck Cancer
}

\author{
Carmen Segrelles ${ }^{1,2,3, *}$, David Contreras ${ }^{1}$, Elena M. Navarro ${ }^{1}$ (iD), Carmen Gutiérrez-Muñoz ${ }^{1}$, \\ Ramón García-Escudero 1,2,3 (iD), Jesús M. Paramio 1,2,3 (iD) and Corina Lorz 1,2,3,* (iD) \\ 1 Molecular Oncology Unit, CIEMAT (ed 70A), Ave Complutense 40, 28040 Madrid, Spain; \\ contreras.david.1987@gmail.com (D.C.); elena.np92@gmail.com (E.M.N.); \\ carmen.gutierrezm@quironsalud.es (C.G.-M.); ramon.garcia@ciemat.es (R.G.-E.); \\ jesusm.paramio@ciemat.es (J.M.P.) \\ 2 Molecular Oncology, University Hospital 12 de Octubre, Research Institute 12 de Octubre i+12, \\ Ave Córdoba s/n, 28041 Madrid, Spain \\ 3 Centro de Investigación Biomédica en Red de Cáncer (CIBERONC), 28029 Madrid, Spain \\ * Correspondence: carmen.segrelles@ciemat.es (C.S.); clorz@ciemat.es (C.L.); \\ Tel.: +34-913467865 (C.S.); +34-914962521 (C.L.); Fax: +34-913466484 (C.S. \& C.L.)
}

Received: 28 May 2018; Accepted: 19 June 2018; Published: 21 June 2018

\begin{abstract}
Head and neck squamous cell carcinoma (HNSCC) is the sixth most common cancer worldwide, and although new therapeutic approaches have been recently evaluated, overall patient survival is still poor. Thus, new effective and selective clinical treatments are urgently needed. An analysis of data from large-scale, high-throughput drug screening cell line projects identified Bosutinib, a Src/Abl inhibitor that is currently used for the treatment of chronic myelogenous leukemia, as a candidate drug to treat HNSCC. Using a panel of HNSCC-derived cell lines, we found that treatment with Bosutinib reduced cell proliferation and induced apoptosis of sensitive cell lines. The drug rapidly inhibited Src and EGFR (epidermal growth factor receptor) phosphorylation, and sensitivity to Bosutinib was correlated with the activation status of EGFR. Similar findings were observed in in vivo xenograft assays using HNSCC derived cells. Moreover, in the presence of mutations in PIK3CA, the combination of Bosutinib with the PI3K $\alpha$ inhibitor Alpelisib showed a synergistic effect. These results suggest that Bosutinib could be a new effective drug for the treatment of HNSCC, particularly in tumors with high EGFR activity. Its combination with Alpelisib could especially benefit patients bearing activating mutations of PIK3CA.
\end{abstract}

Keywords: head and neck cancer; targeted therapies; EGFR inhibitors; Bosutinib; Alpelisib; cancer cell lines

\section{Introduction}

Head and neck cancer arises in the oral and nasal cavities, pharynx and larynx, and in more than $90 \%$ of cases, is of squamous origin. Head and neck squamous cell carcinoma (HNSCC) is the sixth most frequent cancer in the world with an incidence rate of $\sim 600,000$ cases per year [1]. The long-term survival rates are alarmingly low, with half of the patients dying within 5 years [2] and $10-40 \%$ of patients who experience recurrent or metastatic disease dying within a year [3]. The therapeutic options for HNSCC patients are limited. Novel approaches targeting potentially critical pathways are needed to overcome these low survival rates.

Due to the increasing understanding of the molecular mechanisms and basic pathways in the pathogenesis of HNSCC, current research is focused on molecularly-targeted therapies specific for the pathways involved in the carcinogenesis of HNSCC, such as the epidermal growth factor receptor (EGFR) and phosphatidylinositol 3-kinase (PI3K) pathways. The tyrosine kinase epidermal growth 
factor receptor (EGFR) is a well-characterized oncogene in HNSCC. It plays important roles in normal cell growth, lineage determination, repair and functional differentiation. EGFR is frequently altered by activating mutation, amplification and/or overexpression in $\sim 25 \%$ of the tumors [4]. It correlates with poor responses to treatment, increased tumor growth, metastasis and resistance to chemotherapy and radiation therapy [5]. In fact, Cetuximab, a monoclonal, anti-EGFR antibody that binds to EGFR and prevents activation of the downstream signaling pathway, was, until recently, the only approved targeted agent for HNSCC therapy. This medicine can inhibit cell growth and survival and has demonstrated overall survival improvements in clinical trials when combined with radiotherapy or chemotherapy [6,7]. However, the overall increased response to this drug has been lower than initially expected, in part because some patients develop resistance to Cetuximab after an initial benefit. Several studies have identified refractory mechanisms that bypass the inhibition of the EGFR pathway, providing an explanation for the resistance to therapy [8]. Because of this, new drugs targeting the pathway in a different way as well as co-targeting strategies are under investigation. Another cell-growth pathway altered in HNSCC is the PI3K/Akt/mTOR, with PIK3CA (phosphatidylinositol-4,5-bisphosphate 3-kinase catalytic subunit $\alpha$ ) being the most commonly altered gene. This pathway regulates similar processes to those described for EGFR. PIK3CA encodes the catalytic subunit $\alpha$ of class IA PI3K (PI3K $\alpha$, phosphatidylinositol 3-kinase $\alpha$ ) and is affected in $~ 55 \%$ of cases. Activating mutations in PIK3CA have been found in 20\% of HNSCC cases with hot-spot E543K, E545K and H1047R substitutions being the most common $[4,9,10]$.

Based on the analysis of large-scale drug sensitivity screening studies [11], Bosutinib was identified as a candidate drug for HNSCC treatment $[12,13]$. Bosutinib is an orally-active, ATP-binding site competitive inhibitor of Src and Abl kinases. It was approved for the the treatment of Philadelphia chromosome positive chronic myelogenous leukemia by the Food and Drug Administration (FDA) in 2012 [14]. It shares a similar structure to Gefitinib and Erlotinib, which are both FDA-approved EGFR specific tyrosine kinase inhibitors that are under clinical trials for HNSCC [15] (Available online: http:/ / clinicaltrials.gov). A recent study of Src inhibitors confirmed the capability of Bosutinib to inhibit kinases beyond the Src family, directly inhibiting EGFR [16].

In this study, we found that sensitivity to Bosutinib in HNSCC cell lines is dependent on increased EGFR activity. Additionally, we showed that Bosutinib inhibits EGFR activation in vivo in a HNSCC xenograft model. The combination of Bosutinib with the PI3K $\alpha$ inhibitor Alpelisib, which has shown good efficacy and tolerability in several cancers, including HNSCC [17-19], efficiently inhibited both EGFR/ERK and PI3K pathways in HNSCC cell lines. Our results support Bosutinib as a therapy in HNSCC patients, either alone or in combination with Alpelisib in the context of PIK3CA mutations.

\section{Results}

\subsection{Sensitivity of HNSCC Cell Lines to Bosutinib}

We analyzed the sensitivity to Bosutinib in a panel of HNSCC-derived cell lines (Table 1). To cover some of the breadth and complexity of this tumor type, we chose well-characterized cell lines from different head and neck origins, including locoregional (lymph node) metastasis as well as oncogenic alterations commonly found in this type of cancer, such as EGFR overexpression or PIK3CA activating mutation. Our results showed that Bosutinib decreases cell proliferation (Figure 1A) and induces apoptosis in HNSCC cell lines (Figure 1B), which is in agreement with other tumor-derived cell lines $[13,16,20,21]$. The $\mathrm{IC}_{50}$ of three of the six cell lines studied-WSU-HN6, Cal33 and WSU-HN3 - was nearer to the range of peak plasma concentration reached in patients treated with doses of the drug used for cancer therapy [22] (Figure 1A, Table 2); thus, we defined these three cell lines as sensitive, while Detroit562, RPMI2650 and WSU-HN17 were defined as resistant. In Bosutinib-sensitive cell lines, the dose of Bosutinib causing a $75 \%$ decrease in cell viability $\left(\mathrm{IC}_{75}\right.$ as measured by XTT) caused a similar amount of apoptotic cell death as measured by the percentage of cells with $\mathrm{SubG}_{1}$ content in the flow cytometry analysis of the cell cycle (Figure 1B and Table 2). This 
was not the case for the resistant cells, in which the percentage of apoptotic cells was lower, and the decrease in cell viability could be, at least in part, due to an arrest in the progression of the cell cycle.
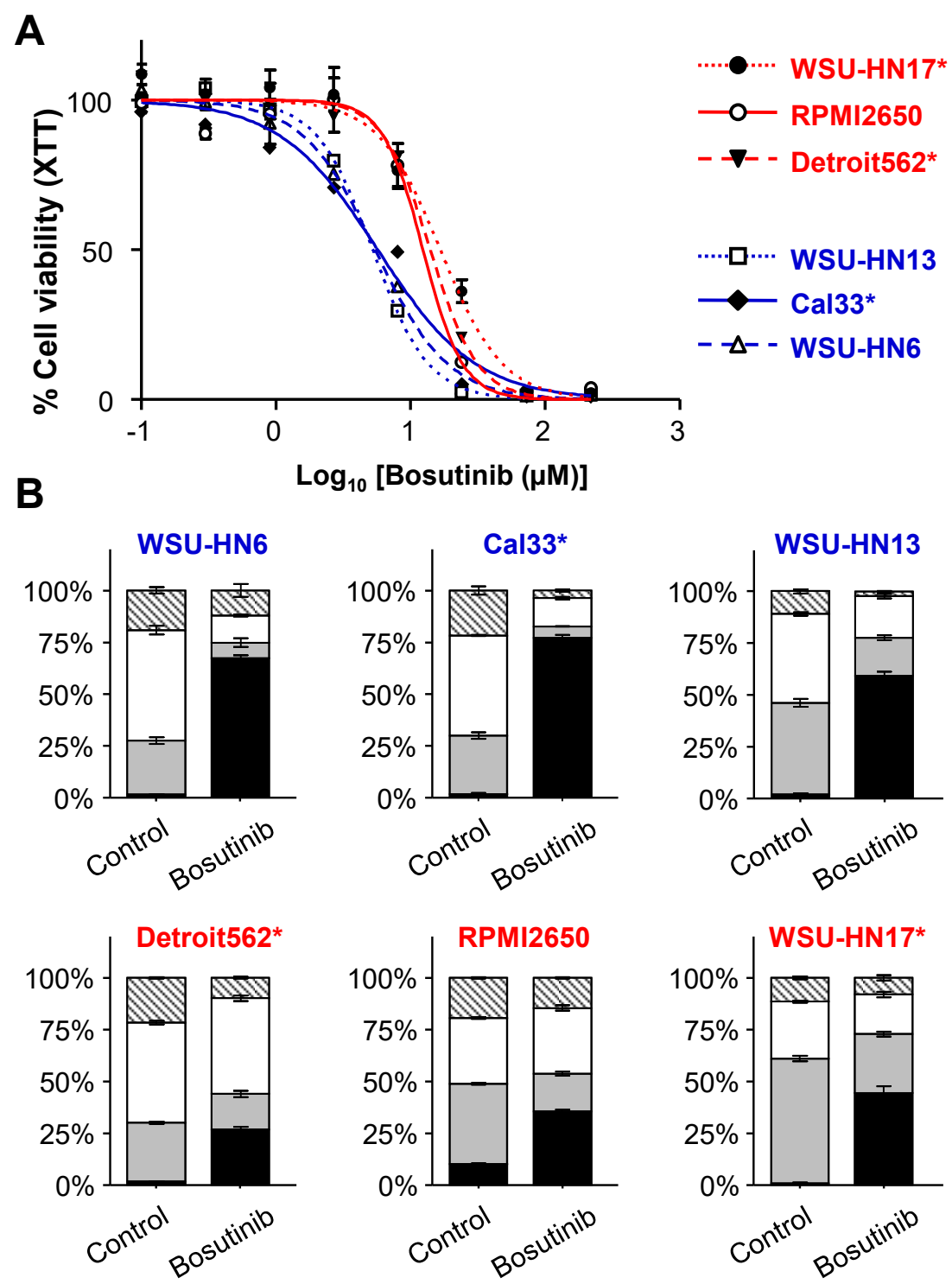

SubG

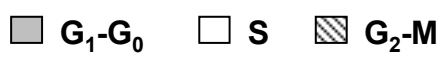

Figure 1. Sensitivity of head and neck squamous cell carcinoma (HNSCC) cell lines to Bosutinib. (A) Cell viability as measured by XTT. Data represent means \pm SEMs from a representative experiment of at least three different experiments for each cell line (each concentration point was replicated six times within each experiment). The cell viability curves (continuous, broken or dotted) for each cell line are a non-linear regression fit of the data (log inhibitor versus normalized response). (B) Cell cycle analysis of Bosutinib-treated HNSCC cell lines. Cells were treated with their corresponding IC $_{75}$ for $24 \mathrm{~h}$ and the cell cycle was analyzed by flow cytometry. The graphs represent the percentage of cells in each phase of the cell cycle. Data represent means \pm SEMs from at least two different experiments (each point was replicated three times within the experiment). ${ }^{*}$ Cell lines with mutations in PIK3CA. In red are cell lines resistant to Bosutinib; in blue are cell lines sensitive to Bosutinib. 
Table 1. HNSCC cell lines.

\begin{tabular}{cccc}
\hline Cell Line & Origin & EGFR & PIK3CA \\
\hline Cal33 & Tongue SCC & $\mathrm{Wt}$ & Mut (H1047R) \\
Detroit562 & Pharynx/pleural effusion metastasis & $\mathrm{Wt}$ & Mut (H1047R) \\
RPMI2650 & Nasal septum metastasis & $\mathrm{Wt}$ & $\mathrm{Wt}$ \\
WSU-HN6 & Base of tongue SCC & $\mathrm{Wt}$ & $\mathrm{Wt}$ \\
WSU-HN13 & Tongue SCC & $\mathrm{Wt}$ & $\mathrm{Wt}$ \\
WSU-HN17 & Lymph node & $\mathrm{Wt}$ & Mut (E542K) \\
\hline
\end{tabular}

WSU: Wayne State University; SCC: squamous cell carcinoma; Mut/Wt: with/without (wild type) mutation in the specified gene

Table 2. Inhibitory concentration (IC) values for Bosutinib and Alpelisib $(\mu \mathrm{M})$, combination index for both drugs at their $\mathrm{IC}_{50}$ and percentage of cells with $\mathrm{SubG}_{1}$ DNA content when treated with the $\mathrm{IC}_{75}$ of Bosutinib.

\begin{tabular}{|c|c|c|c|c|c|c|}
\hline \multirow{2}{*}{ Cell Line } & \multicolumn{3}{|c|}{ Bosutinib } & \multirow{2}{*}{$\begin{array}{l}\text { Alpelisib } \\
\text { IC }_{50}\end{array}$} & \multirow{2}{*}{$\begin{array}{l}\text { Combination } \\
\text { Index } \mathrm{IC}_{50}\end{array}$} & \multirow{2}{*}{$\begin{array}{l}\% \text { of SubG1 Cells } \\
\text { at } \mathrm{IC}_{75} \text { of Bosutinib }\end{array}$} \\
\hline & $\mathrm{IC}_{25}$ & $\mathrm{IC}_{50}$ & $\mathrm{IC}_{75}$ & & & \\
\hline WSU-HN17 * & $14.42 \pm 3.43^{\dagger}$ & $18.56 \pm 2.49 \ddagger$ & $25.02 \pm 2.09 \ddagger$ & - & - & $44.25 \pm 3.09 \ddagger$ \\
\hline RPMI2650 & $10.89 \pm 5.50$ & $14.81 \pm 4.31 \ddagger$ & $23.82 \pm 3.03 \ddagger$ & - & - & $35.53 \pm 0.63 \ddagger$ \\
\hline Detroit562* & $8.27 \pm 0.92$ & $13.25 \pm 0.52 \ddagger$ & $21.52 \pm 1.27 \ddagger$ & $36.84 \pm 6.79$ & 0.25 & $26.76 \pm 1.05 \ddagger$ \\
\hline WSU-HN13 & $5.07 \pm 1.12$ & $6.49 \pm 0.68$ & $8.57 \pm 0.18$ & $35.41 \pm 6.84$ & 0.73 & $59.14 \pm 2.11^{\dagger}$ \\
\hline Cal33 * & $2.98 \pm 0.10$ & $6.09 \pm 0.11$ & $12.76 \pm 0.09$ & $12.67 \pm 2.39$ & 0.63 & $77.17 \pm 1.31 \ddagger$ \\
\hline WSU-HN6 & $2.94 \pm 0.16$ & $5.78 \pm 0.29$ & $11.35 \pm 0.55$ & $35.60 \pm 4.60$ & 0.74 & $67.17 \pm 1.51$ \\
\hline
\end{tabular}

* Cell lines with mutations in PIK3CA. In red are Bosutinib-resistant cell lines. In blue are Bosutinib-sensitive cell lines. IC values are defined as the concentration of drug causing a decrease of $25 \%\left(\mathrm{IC}_{25}\right), 50 \%\left(\mathrm{IC}_{50}\right)$ and $75 \%\left(\mathrm{IC}_{75}\right)$ of cell viability as measured by XTT, respectively. Data are means \pm SEMs from at least three different experiments for each cell line (each concentration point was replicated six times within each experiment). ${ }^{\dagger} p<0.05,{ }^{\ddagger} p<0.01$ compared to the respective value in WSU-HN6 (one-way ANOVA with Bonferroni post-hoc test).

\subsection{Sensitivity to Bosutinib Correlates with Phosphorylated EGFR Protein Levels}

Next, we sought to identify a possible biomarker of sensitivity to Bosutinib in the HNSCC cell lines. We studied the protein and phosphoprotein levels of Src and EGFR in these cells (Figure 2A), and we observed that the relative EGFR activity level (p-EGFR/EGFR) correlated better with sensitivity to the drug (Figure 2B) than that of Src. This is consistent with the observation that Src inhibitors, and in particular Bosutinib, are able to inhibit EGFR activation at levels comparable to the specific EGFR inhibitor Erlotinib [16]. No mutations in the EGFR gene have been reported in the cell lines used in this study. Thus, we studied the copy number variation (CNV) and expression of the EGFR gene (Figure 2C and D upper panel). WSU-HN6, Cal33 and WSU-HN3 Bosutinib-sensitive cell lines showed increased EGFR gene copy number and expression, which are both frequent events in HNSCC [4], as compared to Bosutinib-resistant cell lines. Among the resistant cell lines, the Detroit562 and WSU-HN17 cells showed similar levels of total EGFR protein to Cal33; however, neither of them had amplification of EGFR, and only Detroit562 showed a comparable level of EGFR gene expression. Importantly, EGFR phosphorylation levels were lower in Detroit562 and WSU-HN17 cells compared to Cal33.

PIK3CA alterations, such as mutations and gene amplification and overexpression are common in HNSCC $[4,9,10,23-25]$. Three of the six cell lines studied had activating mutations in this oncogene (Table 1), but the mutations did not seem to yield a differential effect on their sensitivity to Bosutinib (Figure 1). None of the cell lines displayed CNV in PIK3CA (Figure 2C). Also, PIK3CA gene expression was similar among them (Figure 2D, lower panel). 

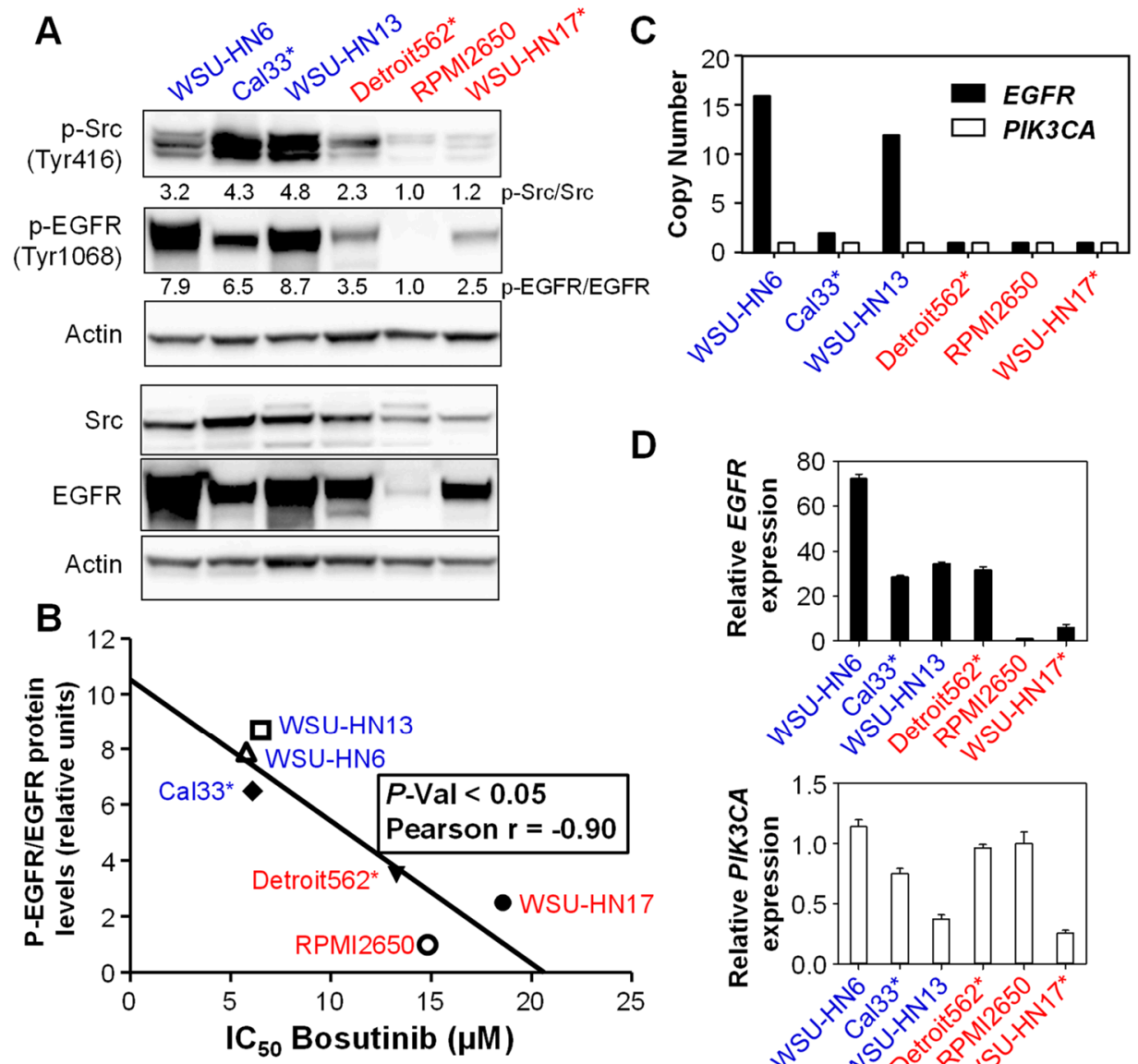

D

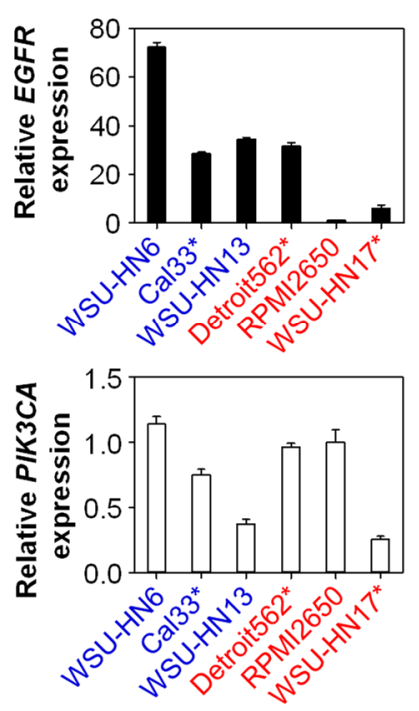

Figure 2. (A) Src and epidermal growth factor receptor (EGFR) phosphorylation was analyzed by Western blot analysis. The numbers indicate the normalized ratio of the phosphorylated form versus the total form. (B) Linear regression of the p-EGFR/EGFR ratio versus sensitivity to Bosutinib ( $\mathrm{IC}_{50}$ ) of the different HNSCC cell lines. EGFR and PIK3CA copy number (C) and mRNA expression (D). * Cell lines with mutations in PIK3CA. In red are cell lines resistant to Bosutinib; in blue are cell lines sensitive to Bosutinib.

\subsection{Bosutinib Effectively Inhibits Src and EGFR Phosphorylation in HNSCC Sensitive Cell Lines and in Xenografts}

To better understand the effect of Bosutinib on HNSCC cell lines, we studied Src and EGFR tyrosine kinase in cells treated with the $\mathrm{IC}_{50}$ dose of the drug at different times. In Bosutinib-sensitive cells, Src activating phosphorylation at Tyr416 was inhibited at early time points (10 $\mathrm{min}$ ) and the inhibition was maintained at later time points (24 h) (Figure 3). Similarly, EGFR phosphorylation at Tyr845 and Tyr1068 was inhibited at early and late time points. It has been well described that Src phosphorylates EGFR on Tyr845 [26]; thus, Bosutinib inhibition of Src activation could mediate this decrease in p-EGFR Tyr845. However, phosphorylation of EGFR at Tyr1048 occurs upon ligand-activated autophosphorylation, and EGFR autokinase activity is not dependent on the Tyr845 phosphorylation status [27]. This suggests that Bosutinib could exert a direct effect on EGFR in HNSCC sensitive cell lines.

To further characterize the effects of Bosutinib in the EGFR blockade, we conducted studies in HNSCC xenografts. Animals bearing Cal33-derived xenografts were treated with Bosutinib or with the EGFR monoclonal antibody drug Cetuximab, as indicated (Figure 4A). Bosutinib inhibited Src and EGFR phosphorylation in the tumors; moreover, Bosutinib-mediated EGFR phosphorylation inhibition was comparable to the effects of Cetuximab (Figure 4B). Both Bosutinib and Cetuximab inhibited 
tumor growth after 10 days of treatment (Figure 4C). An immunohistochemical analysis of the tumors at this time point revealed that both treatments decreased tumor proliferation and increased cell death (Figure 4D).

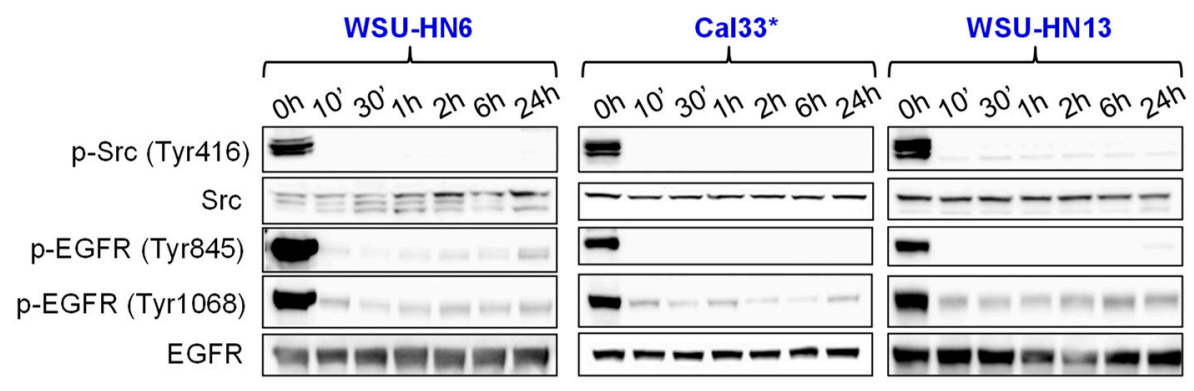

Figure 3. Western blot analysis of Src and EGFR phosphorylation of Bosutinib-sensitive HNSCC cell lines treated with the $\mathrm{IC}_{50}$ of the drug at different time points. ${ }^{*}$ Cell lines with mutations in PIK3CA.

A

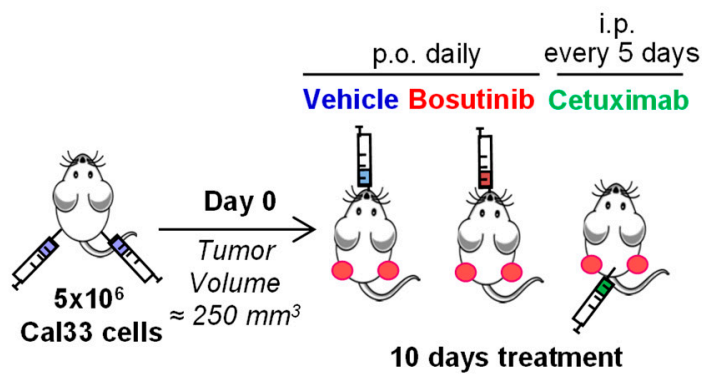

B

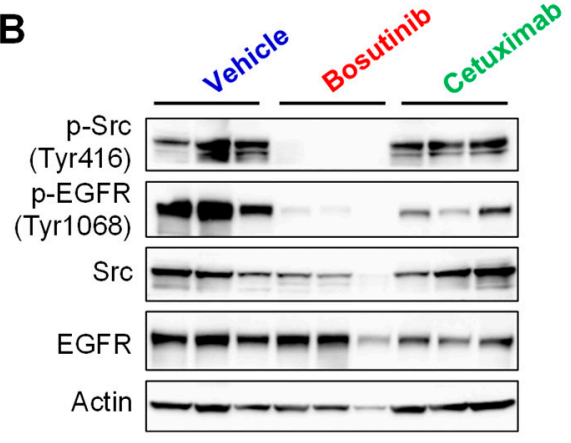

C

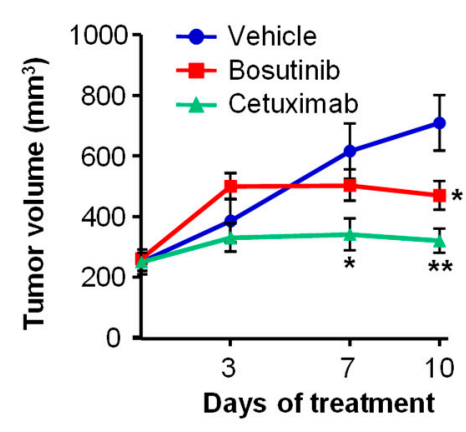

D

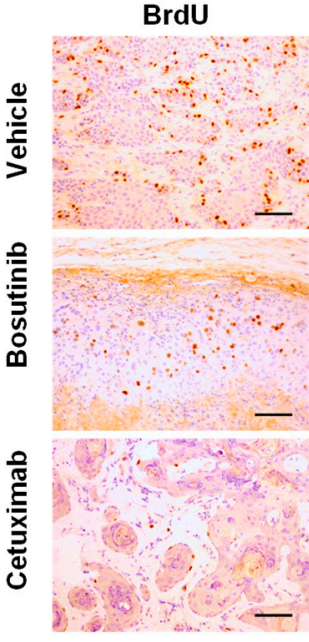

BrdU / K5

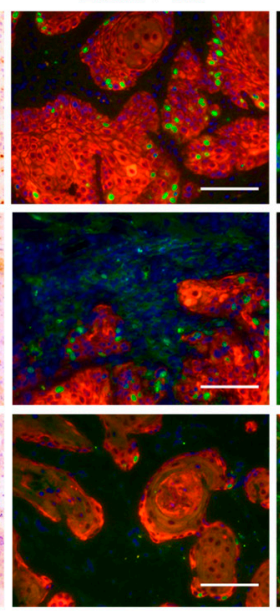

AE1/AE3 / Casp3

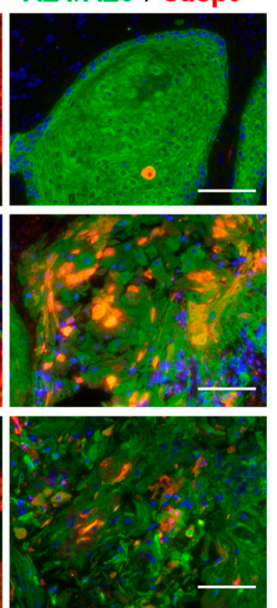

Figure 4. Effect of Bosutunib in HNSCC xenografts. (A) Cal33-derived xenografts were treated as indicated. P.o.: Per Os (oral administration); i.p.: intraperitoneal. (B) Western blot with the indicated antibodies of lysates from tumors treated as specified for 10 days. Each lane is a lysate from a tumor corresponding to the indicated treatment group. (C) Tumor growth of Cal33-derived xenografts treated as indicated $\left(n=10\right.$ per arm). ${ }^{*} p<0.05,{ }^{* *} p<0.01$. (D) Sections from tumors treated as indicated for 10 days were immunostained with BrdU to assess proliferation, the epithelial marker keratin 5 (K5), the pan keratin marker AE1/AE, and the apoptotic marker Caspase 3. Scale bar $100 \mu \mathrm{m}$. 


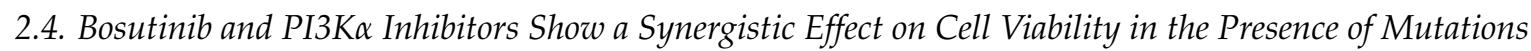
in PIK3CA

Subsequently, we studied the effect of Bosutinib on EGFR downstream signal transducers in sensitive cells. Bosutinib was more effective at inhibiting the phosphorylation of ERK than the phosphorylation of Akt. Phosphorylation of the mTOR downstream target S6 decreased only at later time points (Figure 5A, Supplementary Figure S1A). The combination of Bosutinib with PI3K $\alpha$ inhibitors (Alpelisib or Dactolisib) efficiently inhibited the phosphorylation of ERK, Akt at Ser473, and S6 (Figure 5B, Supplementary Figure S1B,C). In fact, Bosutinib and the combination of Bosutinib and Alpelisib were more effective at inhibiting the downstream signaling of EGFR than Erlotinib alone or together with Alpelisib (Figure 5B, Supplementary Figure S1B,C).
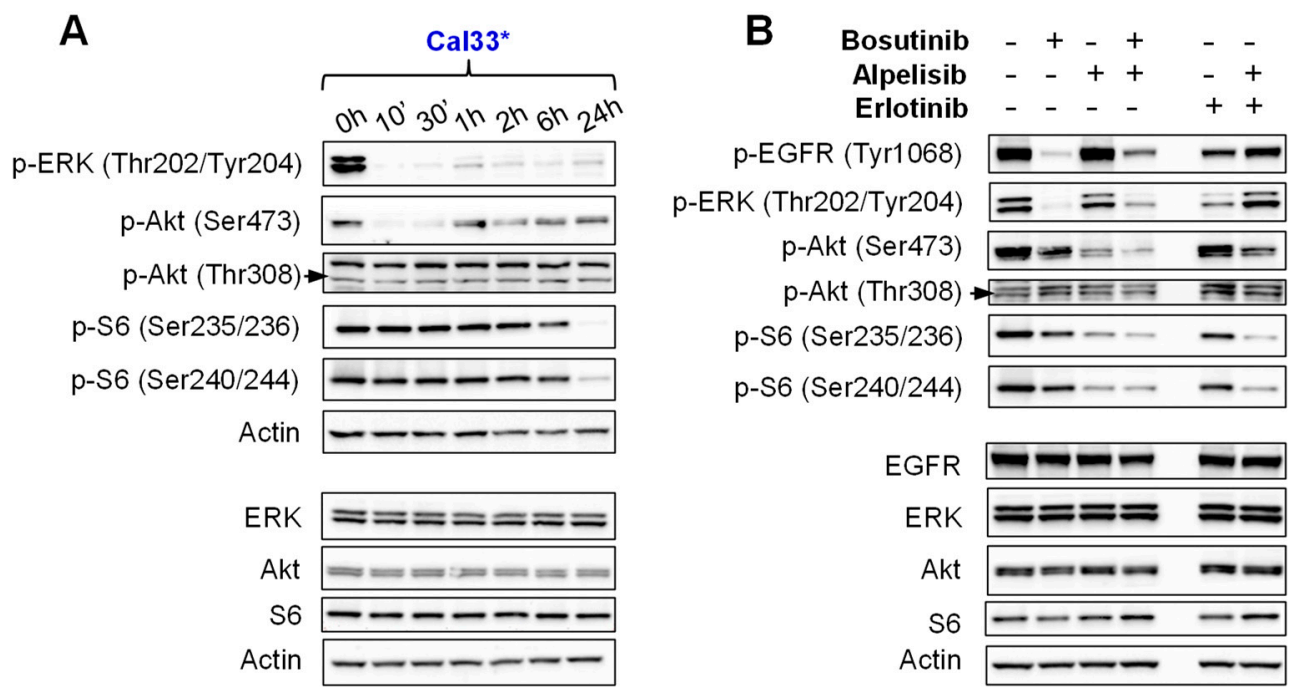

Figure 5. (A) Western blot analysis of ERK, Akt and S6 phosphorylation of Cal33 cells treated with the $\mathrm{IC}_{50}$ of Bosutinib at different time points. (B) Western blot analysis of ERK, Akt and S6 phosphorylation of Cal33 cells treated with Bosutinib $\left(\mathrm{IC}_{50}\right)$, Alpelisib $\left(\mathrm{IC}_{50}\right)$ and Erlotinib $(10 \mu \mathrm{M})$ at $6 \mathrm{~h} .{ }^{*}$ Mutated in PIK3CA. Black arrows indicate p-Akt Thr308.

The combination of targeted therapies to block multiple molecular pathways is an effective strategy to prevent or delay acquired resistance. In this context, we studied the effects of inhibiting both EGFR and PI3K $\alpha$ on HNSCC cell lines. For this purpose, we chose two cell lines wild type for PIK3CA (WSU-HN13 and WSU-HN6) and two with mutation in this gene (Cal33 and Detroit562). The combination of Bosutinib and Alpelisib caused a greater reduction in cell viability than the effect of either drug alone (Figure 6). However, simultaneous inhibition of EFGR and PI3K $\alpha$ resulted in an additive effect or moderate synergism in PIK3CA wild type cells and had a clear synergistic effect in PIK3CA mutant cell lines, irrespective of their sensitivity to Bosutinib (Figure 6B,C, Table 2). 
A

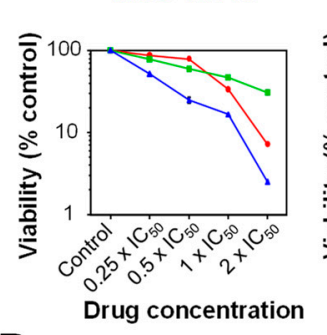

B

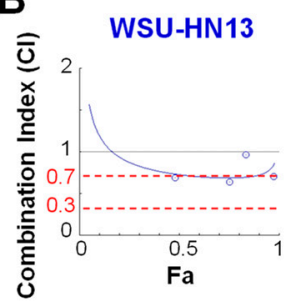

C

\begin{tabular}{|cc|}
\hline $\mathrm{Cl}$ & Description \\
\hline$<0.3$ & Strong synergism \\
$0.3-0.7$ & Synergism \\
$0.7-1$ & Moderate/slight synergism \\
$\approx 1$ & Additive effect \\
$>1$ & Antagonism \\
\hline
\end{tabular}
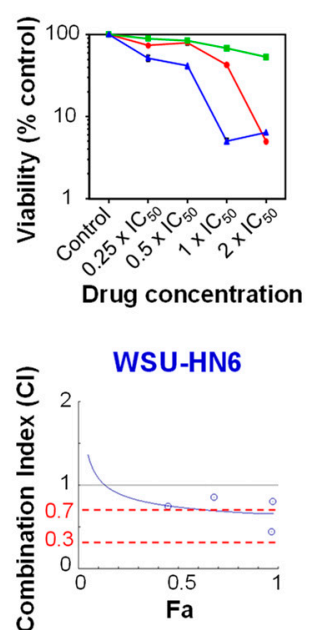

Cal33*
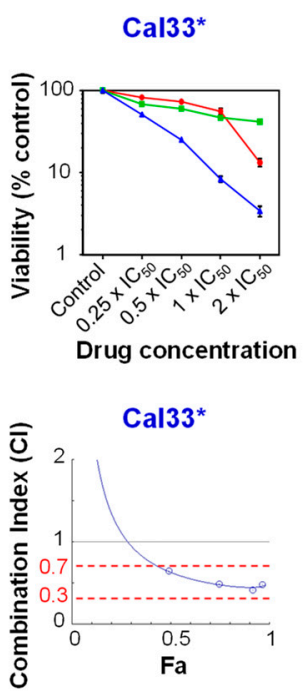

Detroit562*
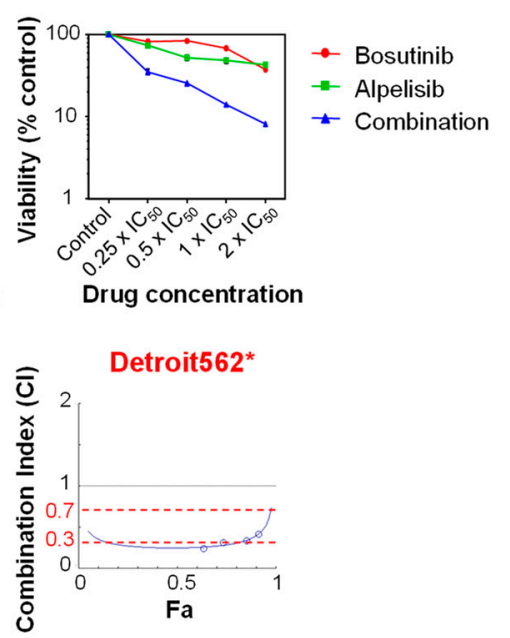

Figure 6. Sensitivity of HNSCC cell lines to Bosutinib in combination with Alpelisib. (A) Cell viability, as measured by XTT of the indicated cell lines treated with Bosutinib, Alpelisib and their combination at the indicated amounts of their $\mathrm{IC}_{50}$ dose. Data represents means \pm SEMs (each concentration point was replicated four times within the experiment). (B) Fa-CI plots to measure the synergism of the combination of two drugs. Fa represents the fraction of the cells affected by the combination of both drugs (at the concentrations indicated in panel A), "0" being $100 \%$ cell survival and " 1 " being $0 \%$ cell survival. The Combination Index (CI) was calculated using Compusyn software and plotted for each combination. (C) Description of the CI values. * Cell lines with mutations in PIK3CA. In red are cell lines resistant to Bosutinib; in blue are cell lines sensitive to Bosutinib.

\section{Discussion}

The World Health Organization predicted that there will be 833,424 new HNSCC cases in 2020, about 100,000 more cases than in 2015. Despite the latest advances in the therapy for this cancer, the mortality rate remains unchanged: $50-55 \%$ (data from Globocan 2012 [1]). There is an urgent need for novel and personalized therapies to improve this outcome. Recent advances in genomic analyses and drug screening techniques have allowed the discovery of new therapeutic targets in several cancers. This is true in the case of EGFR, whose amplification and/or overexpression are frequent events in HNSCC. Although Cetuximab has been approved as an EGFR-targeted medicine for HNSCC treatment, few patients have shown long-term improvement with this treatment. Thus, alternative therapeutic strategies are under investigation. In the present study, we attempted to better define the mechanism of action of Bosutinib in head and neck cancer.

The analysis of large-scale sensitivity studies of pan-cancer cell lines to drugs under clinical and preclinical investigation revealed that Bosutinib is a candidate drug to treat HNSCC [11-13]. These studies suggested that EGFR could be a molecular marker of sensitivity to Bosutinib. It has also been reported that some anti-Src agents, including Bosutinib, could exert a direct effect on EGFR in other cell types [16]. Our findings showed that Bosutinib can inhibit cell growth in a panel of HNSCC cell lines. Bosutinib induced cell death by apoptosis, and the effect was more pronounced in the most sensitive cell lines, indicating that the intrinsic properties and alterations of the cells could affect the mechanism of action of the drug. Treatment with Bosutinib revealed different levels of sensitivity 
among HNSCC cell lines which correlated with the level of the phosphorylated-active form of EGFR. The most sensitive cell lines were those with the highest levels of active EGFR. This could be of help when selecting the group of patients that could benefit most from Bosutinib treatment.

We showed that Bosutinib was very efficient in inhibiting EGFR and Src phosphorylation in HNSCC-sensitive cell lines. Similarly, Bosutinib achieved a complete blockade of EGFR and Src phosphorylation in HNSCC xenografts. Bosutinib decreased tumor growth, and the analysis of the tumors revealed that it reduced proliferation and activated apoptosis of the epithelial cells. In sensitive cell lines, Bosutinib inhibited ERK activation, but the Akt/mTOR pathway remained active. The combination of Bosutinib with the PI3K $\alpha$ inhibitor Alpelisib resulted in the inactivation of both pathways as well as further reduction in cell viability as compared to either drug alone. Cells with alterations (mutation/amplification) in PIK3CA show increased sensitivity to Alpelisib [17]. We observed that the combination of both drugs was most effective in cells with mutations in the PIK3CA gene. Since treatment of PIK3CA mutant and wild type cells with Bosutinib and Alpelisib efficiently inhibited Akt and S6 activation, the differences in their sensitivity levels could be due to a distinct dependence of the cells on the PIK3/Akt/mTOR pathway for their survival.

In summary, the findings of the current work highlight the potential use of Bosutinib as a new drug for the treatment of HNSCC patients, especially for those with activation of the EGFR pathway. In addition, the concomitant administration of Bosutinib and PI3K inhibitors, such as Alpelisib, could be of potential benefit to patients with mutations in PI3K $\alpha$, thus providing a genotype-based treatment. This study provides a first step for future preclinical studies combining Bosutinib and Alpelisib in HNSCC.

\section{Materials and Methods}

\subsection{Cell Lines and Inhibitors}

A panel of six human HNSCC-derived cell lines was selected for the study (Table 1). Cal33, Detroit562, WSU-HN6, WSU-HN13 and WSU-HN17 were kindly provided by J. Silvio Gutkind (Department of Pharmacology and Moores Cancer Center, University of California, San Diego, La Jolla, CA, USA). The mutations in EGFR and PIK3CA were as described in Martin et al. [28] or based on data from Gutkind's lab (WSU-HN17). RPMI2650 was purchased from the American Type Culture Collection (ATCC, Rockville, MD, USA). Cal33, WSU-HN6, WSU-HN13 and WSU-HN17 were cultured in DMEM (Gibco, Thermo Fisher Scientific, Waltham, MA, USA) supplemented with $10 \%$ fetal bovine serum (FBS, Gibco). Detroit562 and RPMI2650 were cultured in EMEM (Lonza, Basel, Switzerland) supplemented with $10 \% \mathrm{FBS}$. All cells were maintained at $37^{\circ} \mathrm{C}$ in an atmosphere of $5 \% \mathrm{CO}_{2}$ and 95\% humidity.

For the in vitro studies, stock solutions of the inhibitors were prepared in DMSO following the manufacturer's guidelines (Selleckchem, Houston, TX, USA) and taking into account the solubility of each compound. The stock concentrations used were $10 \mathrm{mg} / \mathrm{mL}(18.8 \mathrm{mM})$ for the Src/Abl tyrosine kinase inhibitor Bosutinib; $10 \mathrm{mg} / \mathrm{mL}$ (22.6 mM) for the PI3K inhibitor Alpelisib; $1 \mathrm{mg} / \mathrm{mL}$ (2.13 mM) for the PI3K inhibitor Dactolisib and $2.15 \mathrm{mg} / \mathrm{mL}(5 \mathrm{mM})$ for the EGFR inhibitor Erlotinib.

\subsection{Cell Viability Assays and Drug Combination Studies}

Cells were cultured in 96-well plates and, after $24 \mathrm{~h}$ of culturing, they were treated with escalating concentrations of Bosutinib or Alpelisib for $24 \mathrm{~h}$. Cell viability was evaluated with the Colorimetric Assay XTT Cell Proliferation Kit II (Roche, Basel, Switzerland). Background absorbance (medium only) was subtracted, and the data (average of six replicates of each drug concentration) were normalized as percentages of vehicle control. Each experiment was performed at least three times, and each concentration point was replicated six times within each experiment. The concentrations of Bosutinib corresponding to its 25,50 and 75 inhibitory concentrations (IC) $\left(\mathrm{IC}_{25}, \mathrm{IC}_{50}\right.$ and $\left.\mathrm{IC}_{75}\right)$ were calculated with Graph Pad Prism5 software and are shown in Table 2. These values are defined as 
the concentration of drug causing decreases of $25 \%, 50 \%$ and $75 \%$ in cell viability, as measured by XTT, respectively, and they were used for the rest of the experiments in this manuscript (two-drug combination studies, cell cycle analysis and Western blotting).

For the combined viability assays, we followed the method described by Chou and Talalay for two-drug combination studies [29] that calculates a "Combination Index" (CI) to quantitatively depict synergism $(\mathrm{CI}<1)$, additive effects $(\mathrm{CI}=1)$ and antagonism $(\mathrm{CI}>1)$. Briefly, two-fold serial dilutions of the $\mathrm{IC}_{50}$ were performed for each drug alone and their mixture to create $4-5$ concentrations. The combination of the two drugs was performed at a constant ratio. For this purpose, the $\mathrm{IC}_{50}$ concentration for Alpelisib for some of the HNSCC cell lines had to be established (Table 2). The CI was calculated using CompuSyn software (Available online: http:/ /www.combosyn.com/).

\subsection{Cell Cycle Analysis}

The analysis of the cell cycle was performed by flow cytometry. For this purpose, cells were cultured in 12-well plates, and after $24 \mathrm{~h}$, they were treated with their $\mathrm{IC}_{25}, \mathrm{IC}_{50}$ and $\mathrm{IC}_{75}$. After $24 \mathrm{~h}$ of treatment, cells were harvested, washed with PBS and fixed in $70 \%$ ice cold ethanol overnight at $4{ }^{\circ} \mathrm{C}$. The samples were then centrifuged and suspended in PBS containing $2 \mu \mathrm{g} / \mathrm{mL}$ DAPI and $0.05 \%$ NP40 for at least $2 \mathrm{~h}$. Finally, they were analyzed by a Becton Dickinson LSR Fortessa cell analyzer using BD FACSDiva software. Cell debris was excluded from the analysis based on the lower forward scatter/side scatter ratio (FSC-A/SCC-A ratio). Similarly, cell aggregates were excluded based on the FSC/FSC pulse width (FSC-A/FSC-W) and the Violet/Violet pulse width (405Violet450_50-A/405Violet450_50-W) gating. A minimum of 10,000 events were analyzed per sample. Data analyses of the $\mathrm{G}_{0-1}, \mathrm{~S}, \mathrm{G}_{2}-\mathrm{M}$ and the $\mathrm{SubG}_{1}$ (apoptosis) peaks were performed with FlowJo 7.6.5 software using the cell cycle analysis tool. Each experiment was performed at least twice, with 3 replicates of each concentration per experiment.

\subsection{Copy Number Variation (CNV) Analysis}

Genomic DNA was purified using the DNeasy Blood and Tissue Kit (Qiagen, Hilden, Germany) and quantified with a NanoDrop NP 1000 spectrophotometer (Thermo Scientific Nanodrop, Thermo Fisher, Waltham, MA, USA). Gene copy numbers were determined using $10 \mathrm{ng}$ of DNA and the Taq-Man ${ }^{\circledR}$ Copy Number Assay (Applied Biosystems, Foster City, CA, USA), following the manufacturer's instructions. The probes for the target genes were labeled with FAM dye, and the probes for the control region were labeled with VIC dye. The qPCR reaction was run in a 7500 Fast Real-Time PCR System (Applied Biosystems) using the following amplification parameters: $2 \mathrm{~min}$ at $50{ }^{\circ} \mathrm{C}$ and $10 \mathrm{~min}$ at $95^{\circ} \mathrm{C}$, followed by 40 cycles of $15 \mathrm{~s}$ at $95^{\circ} \mathrm{C}$ and $1 \mathrm{~min}$ at $60^{\circ} \mathrm{C}$. The copy number was calculated using the following equation: copy number $=2(\Delta \Delta \mathrm{Ct})$, where $\Delta \Delta \mathrm{Ct}=[\Delta \mathrm{Ct}$ (unknown samples) $-\Delta C C t$ (known control) $]$ and $\Delta C t=[C t(F A M)-C t($ VIC $)]$.

\section{5. mRNA Expression Analysis}

Total RNA was isolated using the miRNeasy Mini Kit (Qiagen) according to the manufacturer's instructions, and genomic DNA was eliminated from the samples by DNase treatment (Rnase-Free Dnase Set, Qiagen). The amount of RNA was quantified as described for genomic DNA. Reverse transcription was performed using the Omniscript RT Kit (Qiagen). qPCR was performed in a 7500 Fast Real-Time PCR System (with the same amplification parameters as described for the CNV assays) using the Power SYBR GREEN PCR Master Mix (Applied Biosystems) and $1 \mu \mathrm{L}$ of cDNA (50 ng) as the template. The reaction efficiency was calculated for each primer combination, and the $\beta$-glucuronidase (GUSB) housekeeping gene was used as the internal reference gene for normalization. The sequences of primers used were as follows: for PIK3CA, forward "GGCTCAAAGACAAGAACAAAGG" and reverse "TCCAGCACATGAACGTGTAAA"; for EGFR, forward "CATGTCGATGGACTTCCAGA" and reverse "GGGACAGCTTGGATCACACT", for GUSB, forward "CGCCCTGCCTATCTGTATTC" and reverse "TCCCCACAGGGAGTGTGTAG". 


\subsection{Western Blotting}

Protein extracts were obtained using a lysis buffer (Hepes $40 \mathrm{mM}$, Triton-100 2\%, $\beta$-glycerophosphate $80 \mathrm{mM}, \mathrm{NaCl} 200 \mathrm{mM}, \mathrm{MgCl}_{2} 40 \mathrm{mM}$, EGTA $20 \mathrm{mM}$ ) supplemented with protease and phosphatase inhibitor cocktails (Roche). Proteins were separated in 4-12\% NuPAGE polyacrylamide gels (Invitrogen, Carlsbad, CA, USA) and then transferred to nitrocellulose membranes (GE Healthcare, Little Chalfont, UK) under wet conditions. Membranes were blocked in 5\% non-fat milk in TBS-Tween (Tris- $\mathrm{HCl} 20 \mathrm{mM}, \mathrm{NaCl} 137 \mathrm{mM}, 0.5 \%$ Tween) and then incubated overnight at $4{ }^{\circ} \mathrm{C}$ with the corresponding primary antibodies in $2.5 \%$ BSA TBS-Tween. Peroxidase-coupled secondary antibodies were used, specific for rabbit IgG (1/5000, GE Healthcare), goat IgG $(1 / 10,000$; Santa Cruz Biotechnology, Dallas, TX, USA) and mouse IgG (1/5000; Jackson, West Grove, PA, USA). The protein bands were detected using Super Signal Western Picoluminiscence Substrate (Pierce, Thermo Fisher) according to the manufacturer's instructions, and they were quantified with Image Lab 5.2.2 software (BioRad, Hercules, CA, USA). To avoid cross-signal contamination in the analysis of protein phosphorylation, equal amounts of each protein lysate were loaded on two different gels and run in parallel. One of the gels was blotted with antibodies against the phosphorylated residues of the protein and the other with antibodies recognizing the total form of the protein. Primary antibodies against the following proteins of phosphoproteins were used: p-Src Tyr416 (clone D49G4), Src (clone 32G6), p-EGFR Tyr1068 (clone D7A5), p-EGFR Tyr845 (clone D63B4), EGFR (clone D38B1), p-Akt Ser473 (clone DE9), p-Akt Thr308 (clone 244F9), p-S6 Ser235 / 236 (clone D68F8), p-S6 Ser240/244 (clone D68F8) and S6 (clone 54D2) from Cell signaling; and Akt (clone N-19), ERK (clone K-23), p-ERK1/2 Thr202/Tyr204 (clone E-4) and $\beta$-actin (clone I-19) from Santa Cruz Biotechnology.

\subsection{HNSCC Tumor-Derived Cell Line Xenograft Mouse Model}

Cal33 cells were trypsinized and suspended in a mixure (2:1) of PBS with Matrigel (BD Biosciences, San Jose, CA, USA). Five million Cal33 cells in a total volume of $150 \mu \mathrm{L}$ of PBS-Matrigel suspension were subcutaneously injected in each flank of $(n=18) 5$-6-week-old immunocompromised nude $(\mathrm{nu} / \mathrm{nu})$ mice (Janvier, Saint-Berthevin, France). After 1 week of injection, tumors were visible. A more detailed description of the Cal33 tumors can be found in Supplementary Figure S2. Tumor growth was followed by measurements twice a week with a digital caliper, and tumor volume was calculated using the formula $0.5 \times$ length $\times$ width ${ }^{2}$ [30]. At a tumor volume of $200-250 \mathrm{~mm}^{3}$, mice were randomized in 3 groups $(n=6)$ to receive the different treatments as follows: vehicle $(0.5 \%$ methylcellulose $0.4 \%$ Tween 80), Bosutinib (Selleckchem) and Cetuximab (Merk Serono, Darmstadt, Germany). Bosutinib was dissolved in the vehicle, and $150 \mathrm{mg} / \mathrm{kg}$ was administrated via oral gavage (per os, p.o.) daily. Cetuximab was injected intraperitoneally (i.p.) every 5 days at a dose of $20 \mathrm{mg} / \mathrm{kg}$. The Bosutinib and Cetuximab concentrations were chosen based on published data [17,31].

After 10 days of treatment, mice were sacrificed, and tumors were collected and preserved in $4 \%$ PBS-buffered formalin or $70 \%$ ethanol for further immunohistochemistry and immunofluorescence analysis, or in liquid nitrogen for Western blot analysis. All animal experiments were conducted in compliance with CIEMAT guidelines, and approved by the Animal Welfare Department of the Comunidad de Madrid (project reference: PROEX 183/15, approved 19/06/2015).

\subsection{Immunohistochemistry and Immunofluorescence}

Samples fixed in formalin or 70\% ethanol were embedded in paraffin wax and sectioned $(5 \mu \mathrm{m})$. Sections were stained with hematoxylin and eosin (H \& E) or processed for immunohistochemistry and immunofluorescence using standard protocols. Mice underwent intraperitoneal injection (i.p.) with bromodeoxyuridine (BrdU; $0.1 \mathrm{mg} / \mathrm{g}$ weight in $0.9 \% \mathrm{NaCl}$; Roche) $1 \mathrm{~h}$ before sacrifice. Immunohistochemistry and immunofluorescence were done with primary antibodies against BrdU (Roche), pan-cytokeratin AE1/AE3 (Abcam, Cambridge, UK), cytokeratin 5 (K5) (Covance, Princeton, NJ, USA) and cleaved caspase-3 (Cell Signaling, Danvers, Massachusetts, USA). Secondary peroxidase 
complexed antibodies (Jackson, Cambridge, UK) were used in the immunohistochemistry studies, while fluorochrome-complexed secondary antibodies (Molecular Probes, Eugene, OR, USA) were used for immunofluorescence. Immunohistochemistries were counterstained with hematoxylin, and immunofluorescences were counterstained with DAPI (Roche).

\subsection{Statistical Analysis}

The $\mathrm{IC}_{50}$ of each cell line was estimated graphically as the concentration of the drug causing 50\% loss in cell viability in XTT assays, using a non-linear regression curve fit in GraphPadPrism 5 software. $\mathrm{IC}_{25}$ and $\mathrm{IC}_{75}$ values were also extrapolated from these curves. Data are shown as means \pm standard errors of the mean (SEM). A Pearson correlation analysis was performed using GraphPadPrism, and $p<0.05$ was considered statistically significant. In order to evaluate the effect of the dual treatment with Bosutinib and Alpelisib, drug combination experiments were designed as described by Chou and Talalay [29] and resultant data were analyzed with CompuSyn software [32]. A synergistic effect was considered when the Combination Index (CI) was below 0.7.

Supplementary Materials: Supplementary materials can be found at http:/ / www.mdpi.com/1422-0067/19/7/ $1824 / s 1$.

Author Contributions: C.S., R.G-E., J.M.P. and C.L. conceived and designed the experiments; C.S., D.C. E.M.N., C.G.-M. and C.L. performed the experiments; C.S. and C.L. analyzed the data; C.L. wrote the paper.

Founding: This work was supported by FEDER cofounded MINECO [grant number SAF2015-66015-R] and ISCIII [grant numbers CB16/12/00228, PIE15/00076].

Acknowledgments: We thank Marta Dueñas for help with the mRNA expression and CNV analyses. We thank Beatriz Castelo and José Luis Cebrián from the La Paz University Hospital Madrid for Cetuximab. We thank Norman Feltz for proofreading the article.

Conflicts of Interest: The authors declare no conflict of interest.

\section{Abbreviations}

$\begin{array}{ll}\text { EGFR } & \text { Epidermal growth factor receptor } \\ \text { HNSCC } & \text { Head and neck squamous cell carcinoma } \\ \text { PIK3CA } & \text { Phosphatidylinositol-4,5-bisphosphate 3-kinase catalytic subunit } \alpha \\ \text { PI3K } \alpha & \text { Phosphatidylinositol 3-kinase } \alpha \\ \text { P.o. } & \text { Per Os (oral administration) } \\ \text { I.p. } & \text { Intraperitoneal }\end{array}$

\section{References}

1. Ferlay, J.; Soerjomataram, I.; Dikshit, R.; Eser, S.; Mathers, C.; Rebelo, M.; Parkin, D.M.; Forman, D.; Bray, F. Cancer incidence and mortality worldwide: Sources, methods and major patterns in GLOBOCAN 2012. Int. J. Cancer 2015, 136, E359-E386. [CrossRef] [PubMed]

2. Leemans, C.R.; Braakhuis, B.J.; Brakenhoff, R.H. The molecular biology of head and neck cancer. Nat. Rev. Cancer 2011, 11, 9-22. [CrossRef] [PubMed]

3. Leon, X.; Hitt, R.; Constenla, M.; Rocca, A.; Stupp, R.; Kovacs, A.F.; Amellal, N.; Bessa, E.H.; Bourhis, J. A retrospective analysis of the outcome of patients with recurrent and/or metastatic squamous cell carcinoma of the head and neck refractory to a platinum-based chemotherapy. Clin. Oncol. 2005, 17, 418-424. [CrossRef]

4. Cancer Genome Atlas Network. Comprehensive genomic characterization of head and neck squamous cell carcinomas. Nature 2015, 517, 576-582.

5. Rabinowits, G.; Haddad, R.I. Overcoming resistance to EGFR inhibitor in head and neck cancer: A review of the literature. Oral. Oncol. 2012, 48, 1085-1089. [CrossRef] [PubMed]

6. Vermorken, J.B.; Mesia, R.; Rivera, F.; Remenar, E.; Kawecki, A.; Rottey, S.; Erfan, J.; Zabolotnyy, D.; Kienzer, H.R.; Cupissol, D.; et al. Platinum-based chemotherapy plus cetuximab in head and neck cancer. N. Engl. J. Med. 2008, 359, 1116-1127. [CrossRef] [PubMed] 
7. Bonner, J.A.; Harari, P.M.; Giralt, J.; Azarnia, N.; Shin, D.M.; Cohen, R.B.; Jones, C.U.; Sur, R.; Raben, D.; Jassem, J.; et al. Radiotherapy plus cetuximab for squamous-cell carcinoma of the head and neck. N. Engl. J. Med. 2006, 354, 567-578. [CrossRef] [PubMed]

8. Vlacich, G.; Coffey, R.J. Resistance to EGFR-targeted therapy: A family affair. Cancer Cell 2011, 20, 423-425. [CrossRef] [PubMed]

9. Lawrence, M.S.; Stojanov, P.; Mermel, C.H.; Robinson, J.T.; Garraway, L.A.; Golub, T.R.; Meyerson, M.; Gabriel, S.B.; Lander, E.S.; Getz, G. Discovery and saturation analysis of cancer genes across 21 tumour types. Nature 2014, 505, 495-501. [CrossRef] [PubMed]

10. García-Escudero, R.; Segrelles, C.; Dueñas, M.; Pombo, M.; Ballestín, C.; Alonso-Riaño, M.; Nenclares, P.; Álvarez-Rodríguez, R.; Sánchez-Aniceto, G.; Ruíz-Alonso, A.; López-Cedrún, J.L. Overexpression of PIK3CA in head and neck squamous cell carcinoma is associated with poor outcome and activation of the YAP pathway. Oral. Oncol. 2018, 79, 55-63. [CrossRef] [PubMed]

11. Garnett, M.J.; Edelman, E.J.; Heidorn, S.J.; Greenman, C.D.; Dastur, A.; Lau, K.W.; Greninger, P.; Thompson, I.R.; Luo, X.; Soares, J.; et al. Systematic identification of genomic markers of drug sensitivity in cancer cells. Nature 2012, 483, 570-575. [CrossRef] [PubMed]

12. Nichols, A.C.; Black, M.; Yoo, J.; Pinto, N.; Fernandes, A.; Haibe-Kains, B.; Boutros, P.C.; Barrett, J.W. Exploiting high-throughput cell line drug screening studies to identify candidate therapeutic agents in head and neck cancer. BMC Pharmacol. Toxicol. 2014, 15, 66. [CrossRef] [PubMed]

13. Lee, B.K.B.; Tiong, K.H.; Chang, J.K.; Liew, C.S.; Rahman, Z.A.A.; Tan, A.C.; Khang, T.F.; Cheong, S.C. DeSigN: Connecting gene expression with therapeutics for drug repurposing and development. BMC Genom. 2017, 18, 934. [CrossRef] [PubMed]

14. Rusconi, F.; Piazza, R.; Vagge, E.; Gambacorti-Passerini, C. Bosutinib: A review of preclinical and clinical studies in chronic myelogenous leukemia. Expert Opin. Pharm. 2014, 15, 701-710. [CrossRef] [PubMed]

15. Fung, C.; Grandis, J.R. Emerging drugs to treat squamous cell carcinomas of the head and neck. Expert Opin. Emerg. Drugs 2010, 15, 355-373. [CrossRef] [PubMed]

16. Formisano, L.; D'Amato, V.; Servetto, A.; Brillante, S.; Raimondo, L.; Di Mauro, C.; Marciano, R.; Orsini, R.C.; Cosconati, S.; Randazzo, A.; et al. Src inhibitors act through different mechanisms in Non-Small Cell Lung Cancer models depending on EGFR and RAS mutational status. Oncotarget 2015, 6, 26090-26103. [CrossRef] [PubMed]

17. Elkabets, M.; Pazarentzos, E.; Juric, D.; Sheng, Q.; Pelossof, R.A.; Brook, S.; Benzaken, A.O.; Rodon, J.; Morse, N.; Yan, J.J.; et al. AXL mediates resistance to PI3K $\alpha$ inhibition by activating the EGFR/PKC/mTOR axis in head and neck and esophageal squamous cell carcinomas. Cancer Cell 2015, 27, 533-546. [CrossRef] [PubMed]

18. Fritsch, C.; Huang, A.; Chatenay-Rivauday, C.; Schnell, C.; Reddy, A.; Liu, M.; Kauffmann, A.; Guthy, D.; Erdmann, D.; De Pover, A.; et al. Characterization of the novel and specific PI3K $\alpha$ inhibitor NVP-BYL719 and development of the patient stratification strategy for clinical trials. Mol. Cancer Ther. 2014, 13, 1117-1129. [CrossRef] [PubMed]

19. Wong, C.H.; Ma, B.B.; Cheong, H.T.; Hui, C.W.; Hui, E.P.; Chan, A.T. Preclinical evaluation of PI3K inhibitor BYL719 as a single agent and its synergism in combination with cisplatin or MEK inhibitor in nasopharyngeal carcinoma (NPC). Am. J. Cancer Res. 2015, 5, 1496-1506. [PubMed]

20. Nam, A.R.; Kim, J.W.; Park, J.E.; Bang, J.H.; Jin, M.H.; Lee, K.H.; Kim, T.Y.; Han, S.W.; Im, S.A.; Kim, T.Y.; et al. Src as a Therapeutic Target in Biliary Tract Cancer. Mol. Cancer Ther. 2016, 15, 1515-1524. [CrossRef] [PubMed]

21. Vojvodic, M.; Hansford, L.; Morozova, O.; Blakely, K.; Taylor, P.; Fathers, K.; Moffat, J.; Marra, M.; Smith, K.; Moran, M.; et al. A phosphoproteomics approach to identify candidate kinase inhibitor pathway targets in lymphoma-like primary cell lines. Curr. Drug Discov. Technol. 2013, 10, 283-304. [CrossRef] [PubMed]

22. U.S. Food \& Drug Administration. Available online: https:/ /www.accessdata.fda.gov/drugsatfda_docs / label/2012/203341lbl.pdf (accessed on 19 June 2018).

23. Stransky, N.; Egloff, A.M.; Tward, A.D.; Kostic, A.D.; Cibulskis, K.; Sivachenko, A.; Kryukov, G.V.; Lawrence, M.S.; Sougnez, C.; McKenna, A.; et al. The mutational landscape of head and neck squamous cell carcinoma. Science 2011, 333, 1157-1160. [CrossRef] [PubMed] 
24. Pickering, C.R.; Zhang, J.; Yoo, S.Y.; Bengtsson, L.; Moorthy, S.; Neskey, D.M.; Zhao, M.; Alves, M.V.O.; Chang, K.; Drummond, J.; et al. Integrative genomic characterization of oral squamous cell carcinoma identifies frequent somatic drivers. Cancer Discov. 2013, 3, 770-781. [CrossRef] [PubMed]

25. Lui, V.W.; Hedberg, M.L.; Li, H.; Vangara, B.S.; Pendleton, K.; Zeng, Y.; Lu, Y.; Zhang, Q.; Du, Y.; Gilbert, B.R.; et al. Frequent mutation of the PI3K pathway in head and neck cancer defines predictive biomarkers. Cancer Discov. 2013, 3, 761-769. [CrossRef] [PubMed]

26. Sato, K. Cellular functions regulated by phosphorylation of EGFR on Tyr845. Int. J. Mol. Sci. 2013, 14, 10761-10790. [CrossRef] [PubMed]

27. Tice, D.A.; Biscardi, J.S.; Nickles, A.L.; Parsons, S.J. Mechanism of biological synergy between cellular Src and epidermal growth factor receptor. Proc. Natl. Acad. Sci. USA 1999, 96, 1415-1420. [CrossRef] [PubMed]

28. Martin, D.; Abba, M.C.; Molinolo, A.A.; Vitale-Cross, L.; Wang, Z.; Zaida, M.; Delic, N.C.; Samuels, Y.; Lyons, J.G.; Gutkind, J.S. The head and neck cancer cell oncogenome: A platform for the development of precision molecular therapies. Oncotarget 2014, 5, 8906-8923. [CrossRef] [PubMed]

29. Chou, T.C.; Talalay, P. Quantitative analysis of dose-effect relationships: The combined effects of multiple drugs or enzyme inhibitors. Adv. Enzyme. Regul. 1984, 22, 27-55. [CrossRef]

30. Tomayko, M.M.; Reynolds, C.P. Determination of subcutaneous tumor size in athymic (nude) mice. Cancer Chemother. Pharmacol. 1989, 24, 148-154. [CrossRef] [PubMed]

31. Golas, J.M.; Lucas, J.; Etienne, C.; Golas, J.; Discafani, C.; Sridharan, L.; Boghaert, E.; Arndt, K.; Ye, F.; Boschelli, D.H.; et al. SKI-606, a Src/Abl inhibitor with in vivo activity in colon tumor xenograft models. Cancer Res. 2005, 65, 5358-5364. [CrossRef] [PubMed]

32. Chou, T.C. Theoretical basis, experimental design, and computerized simulation of synergism and antagonism in drug combination studies. Pharmacol. Rev. 2006, 58, 621-681. [CrossRef] [PubMed]

(C) 2018 by the authors. Licensee MDPI, Basel, Switzerland. This article is an open access article distributed under the terms and conditions of the Creative Commons Attribution (CC BY) license (http:/ / creativecommons.org/licenses/by/4.0/). 